\title{
Subdivisions and Anatomical Boundaries of the Subthalamic Nucleus
}

\author{
Anneke Alkemade \\ Cognitive Science Center Amsterdam, University of Amsterdam, Amsterdam, 1018 WS Netherlands \\ Review of Haynes and Haber.
}

The subthalamic nucleus (STN) lies deep within the brain and plays a central role in both the direct and indirect pathway of the basal ganglia (Temel et al., 2005). The nucleus itself does not degenerate in patients with Parkinson's disease, but it is an important target for deep brain stimulation (DBS) in the treatment of this neurodegenerative condition. Immunocytochemical studies provide evidence for dopamine signaling within the nucleus (Hedreen, 1999), but it is unclear whether the dopaminergic input to the STN is affected in Parkinson's disease. Although targeting the STN with DBS effectively relieves severe motor symptoms, it may also produce neuropsychiatric side effects including apathy, compulsive behavior, hypersexuality, cognitive dysfunction, clinical depression, and suicidal ideation (Temel et al., 2005). These side effects have been attributed to the unwanted stimulation of putative nonmotor subdivisions of the STN.

Current hypotheses suggest a tripartite division of the STN with a dorsolateral motor area, a central cognitive/associative area, and a limbic medial tip (Parent and Hazrati, 1995). Recent developments in in vivo imaging techniques, such as 7 tesla (T) MRI have greatly improved the ability to visualize the

Received March 25, 2013; revised April 19, 2013; accepted April 28, 2013. I thank Dr. B. U. Forstmann for valuable discussions.

Correspondence should be addressed to Dr. Anneke Alkemade, Cognitive Science Center Amsterdam, University of Amsterdam, Nieuwe Achtergracht 129, 1018 WS Amsterdam, The Netherlands. E-mail: jmalkemade@gmail.com.

DOI:10.1523/JNEUROSCI.1266-13.2013

Copyright $\odot 2013$ the authors $\quad 0270-6474 / 13 / 339233-02 \$ 15.00 / 0$
STN and investigate putative neuroanatomical subdivisions in young and aging humans (Forstmann et al., 2012; Keuken et al., 2013). Eventually, 7T MRI might be implemented in clinical practice to guide stereotactic implantation of electrical stimulators in Parkinson's patients. Together with further improvements of the DBS stimulators, it is likely that specific subparts of the nucleus can be targeted more reliably. These technical developments call for a clear anatomical delineation of the STN's putative subdivisions to identify surgical targets.

A well executed and extensive viral tracing study recently published in The Journal of Neuroscience by Haynes and Haber (2013) addressed whether the primate STN consists of three anatomically and functionally distinct subdivisions by mapping connectivity profiles. The authors investigated cortical projections to the STN and collected data from an impressive number of 43 macaques, totaling 48 cortical injection sites with anterograde or bidirectional tracers. The application and quantification of viral tracing methods is technically challenging as evidenced by the number of tracings (16 of 48) that were deemed not suitable for modeling for various reasons. Twentyone of 48 injection sites were analyzed. Despite the large number of total injections, therefore, conclusions per STN projection site were based on a limited number of observations (ranging between 2 and 4), for each cortical area. Therefore caution should be used interpreting these findings. The authors discussed method- ological considerations, and decided not to quantify projection volumes and densities in view of the observed variation between injections.

Haynes and Haber (2013) found that the limbic medial tip of the STN was densely innervated by projections from the dorsal anterior cingulate (dACC), which extended over the medial border of the STN. Surprisingly, projections from the ventromedial prefrontal cortex/orbitofrontal cortex (vmPFC/OFC) were located mainly in the lateral hypothalamic area outside of the conventional medial borders of the STN, concentrated near the anterior part of the STN. Projections from motor area 1 (M1) and area 6 were located in the dorsolateral part of the STN. The injection sites were small, and only parts of the frontal cortex were sampled. The authors therefore do not exclude the possibility that STN projection fields of each cortical region may have been larger than reported (Haynes and Haber, 2013).

The extension of vmPFC/OFC projections over the medial borders of the STN is interesting. As the authors pointed out, the medial border of the STN is difficult to delineate. Despite this, the authors claim that the majority of vmPFC terminal fields were located medially to the tip of the STN, in the general area of the lateral hypothalamus ( $\mathrm{LH})$. Based on this finding the authors propose that a component of the LH might be considered as the limbic cone of the STN (Haynes and Haber, 2013). This conclusion, however, remains speculative, and no double labeling exper- 
iments were performed to further identify these neurons as either belonging to the STN or LH. An alternative possibility is that these neurons belong to the LH and that the LH plays a role in limbic function. A role for the LH in responding to emotions is supported by observations in narcolepsy with cataplexy patients (Kornum et al., 2011). Strong emotions are associated with a sudden loss of muscle tone in these patients, which is caused by a specific loss of hypocretin/orexin neurons in the lateral hypothalamic area. Finally, the ontogeny of the neurons labeled in this medial projection area is unknown and further research is needed to clarify their exact identity either belonging to the STN or LH.

Importantly, the viral tracing technique used by Haynes and Haber (2013) produced a labeling pattern within the STN that did not allow clear anatomical delineation of subdivisions. Different functional territories, although topologically organized, were not completely segregated. It appeared that neuronal populations receiving input from limbic, cognitive, and motor areas showed pronounced topological overlap, and the size of projection fields may have been underestimated because of the use of small injection sites (Haynes and Haber, 2013). Topological overlap in the STN of projections from distinct cortical areas is corroborated by a recent study using diffusion weighted imaging in young human participants (Lambert et al., 2012). In that study, left and right STNs of 12 healthy participants were investigated. Support for the existence of three functional subdivisions was found in 14 of 24 STNs. A more detailed investigation of the connectivity profile revealed that a substantial number of voxels met the criteria for being part of more than one STN subdivision (Lambert et al., 2012; supplemental material). This is in line with the findings of topologically overlapping functional regions within the STN as proposed by Haynes and

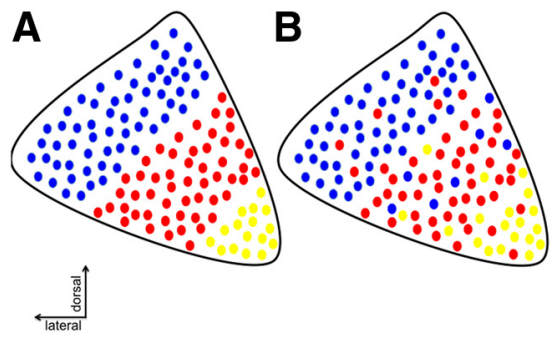

Figure 1. Topological organization of the STN. A, Schematic representation of anatomically distinct functional subdivisions of the STN. $\boldsymbol{B}$, Alternative topological organization of the STN as supported by Haynes and Haber (2013). Yellow dots represent neurons from the limbic STN, red dots represent neurons from the associative/cognitive STN, and blue dots represent neurons from the motor circuit.

Haber (2013), rather than the existence of distinct anatomical subdivisions. Although highly speculative, the topological overlap may also explain why Lambert and colleagues were not able to identify three subdivisions in nearly half of the studied STNs.

The studies of Haynes and Haber (2013) and Lambert et al. (2012) have significant implications for the current views on STN subdivisions. Although topological differences are observed, neither study provides unequivocal evidence for anatomically delineated STN anatomical subdivisions, but argue for a topological organization without strict anatomical borders (Fig. 1). In addition, these findings suggest that more precise placement of electrical probes within subregions of the STN might reduce side effects resulting from unwanted stimulation of the cognitive or limbic subparts; but, it is unlikely that side effects can be prevented completely, given the topological overlap between functional areas within the STN, as well as the spread of electrical currents within the tissue.

In conclusion, the study by Haynes and Haber (2013) has provided important information on the overlap of functional projection regions within the STN, which is corroborated by recent diffusion weighted imaging studies in humans (Lambert et al., 2012). These findings are relevant for further optimization of surgical treatment of Parkinson's disease.

\section{References}

Forstmann BU, Keuken MC, Jahfari S, Bazin PL, Neumann J, Schäfer A, Anwander A, Turner R (2012) Cortico-subthalamic white matter tract strength predicts interindividual efficacy in stopping a motor response. Neuroimage 60:370-375. CrossRef Medline

Haynes WI, Haber SN (2013) The organization of prefrontal-subthalamic inputs in primates provides an anatomical substrate for both functional specificity and integration: Implications for basal ganglia models and deep brain stimulation. J Neurosci 33:4804-4814. CrossRef Medline

Hedreen JC (1999) Tyrosine Hydroxylaseimmunoreactive elements in the human globus pallidus and subthalamic nucleus. J Comp Neurol 409:400-410. CrossRef Medline

Keuken MC, Bazin PL, Schäfer A, Neumann J, Turner R, Forstmann BU (2013) Ultra-high 7T MRI of structural age-related changes of the subthalamic nucleus. J Neurosci 33: 4896-4900. CrossRef Medline

Kornum BR, Faraco J, Mignot E (2011) Narcolepsy with hypocretin/orexin deficiency, infections and autoimmunity of the brain. Curr Opin Neurobiol 21:897-903. CrossRef Medline

Lambert C, Zrinzo L, Nagy Z, Lutti A, Hariz M, Foltynie T, Draganski B, Ashburner J, Frackowiak R (2012) Confirmation of functional zones within the human subthalamic nucleus: patterns of connectivity and sub-parcellation using diffusion weighted imaging. Neuroimage 60:83-94. CrossRef Medline

Parent A, Hazrati LN (1995) Functional anatomy of the basal ganglia. II. The place of subthalamic nucleus and external pallidum in basal ganglia circuitry. Brain Res Brain Res Rev 20:128-154. CrossRef Medline

Temel Y, Blokland A, Steinbusch HW, VisserVandewalle V (2005) The functional role of the subthalamic nucleus in cognitive and limbic circuits. Prog Neurobiol 76:393-413. CrossRef Medline 